DOI:

10.1038/nrg2259

\title{
The microarray revolution
}

Imagine attempting to measure the expression level of every gene in the genome one at a time. Now imagine assaying thousands of genes all at once. It was the advent of microarray technology that made possible this leap from low to high throughput, allowing researchers to ask questions on a scale that was previously unattainable.

The landmark study that showed that DNA could be made into microarrays was published in 1991 by Stephen Fodor and colleagues at the Affymax Research Institute. They showed that a diverse set of oligonucleotides could be chemically synthesized on a glass slide through photolithography, a process using precisely aimed beams of light to direct chemical reactions to specific spots. This allows for miniaturization because the density of spots is limited only by the diffraction of light.

A paper published in 1995 by Patrick Brown and colleagues at Stanford University brought attention to the exciting potential of microarray technology. They used a microarray of 45 Arabidopsis complementary DNAs to which they hybridized fluorescently-labelled total cellular messenger RNA (mRNA). The intensity of fluorescence at a spot reflected the amount of mRNA hybridized, which in turn reflected the level of that particular mRNA in the initial sample. This paper showed for the first time that the expression of many genes in a small sample could be quantitatively monitored in parallel.

Microarray-based expression profiling has useful applications

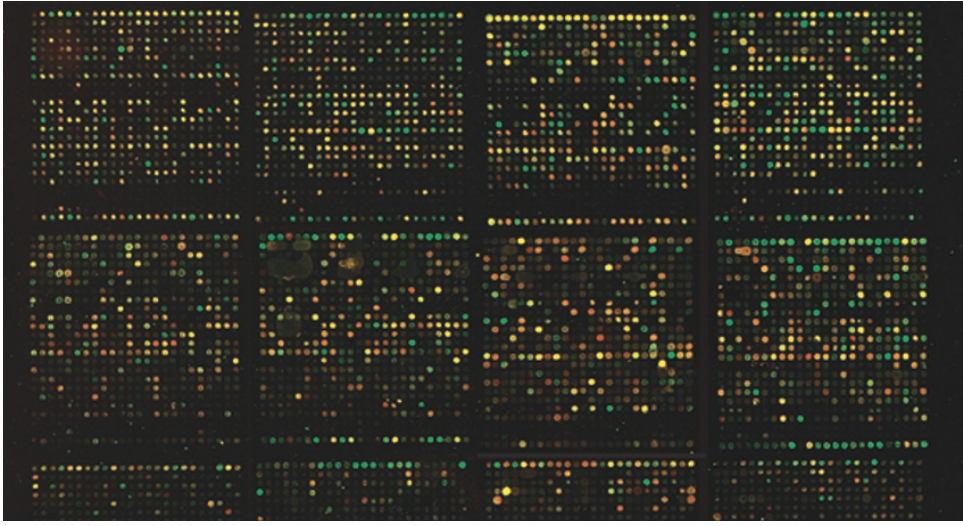

in medical research, as it reveals molecular portraits of gene expression in disease states. Yet the utility of microarrays is not limited to measuring gene expression. Once the technology became established, researchers began to use microarrays to measure other important biological phenomena. For example, microarrays are being used to genotype single-nucleotide polymorphisms by hybridizing the DNA of individuals to arrays of oligonucleotides representing different polymorphic alleles. An application called array-comparative genomic hybridization is being used to detect genomic structural variation, such as segments of the genome that have varying numbers of copies in different individuals; this is accomplished by hybridizing the total genomic DNA to an array of oligonucleotides representing DNA fragments distributed evenly throughout the genome. Epigenetic marks associated with certain areas of the genome, such as chromatin modifications, are being profiled using microarrays in an application called ChIP-chip (see Milestone 14).

Microarray technology has grown from a pioneering method applied by innovators at the cutting edge to a ubiquitous technique that has allowed researchers to investigate 'big-picture' questions in biology. This miniaturized technology has brought about a major revolution.

Emily Niemitz, Associate Editor, Nature Genetics

ORIGINAL RESEARCH PAPERS Fodor, S. P. et al. Light-directed, spatially addressable parallel chemical synthesis. Science $\mathbf{2 5 1}$,

767-773 (1991) | Schena, M., Shalon, D., Davis, R. W. \& Brown, P. O. Quantitative monitoring of gene expression patterns with a complementary DNA microarray. Science 270, 467-470 (1995) FURTHER READING Southern, E. M. et al. Analyzing and comparing nucleic acid sequences by hybridization to arrays of oligonucleotides: evaluation using experimental models. Genomics 13, 1008-1017 (1992)|Pease, A. C. et al. Light-generated oligonucleotide arrays for rapid DNA sequence analysis. Proc. Natl Acad. Sci. USA 91, 5022-5026 (1994)|Hoheisel, J. D. Microarray technology: beyond transcript profiling and genotype analysis. Nature Rev. Genet. 7; 200-210 (2006)|Allison, D. B., Cui, X., Page, G. P. \& Sabripour, M. Microarray data analysis: from disarray to consolidation and consensus. Nature Rev. Genet. 7, 55-65 (2006)| Fan, J. B., Chee, M. S., Gunderson, K. L. Highly parallel genomic assays. Nature Rev. Genet. 7 , 632-644 (2006) 